\title{
Composición corporal y trastornos respiratorios del sueño en escolares de Valdivia, Chile
}

\author{
Relationship between body composition and sleep-disordered breathing \\ in schoolchildren from Valdivia, Chile
}

\author{
Valentina Da Bovea, Claudia Papamichail ${ }^{\mathrm{b}}$, Romina Vera ${ }^{\mathrm{b}}$, \\ Ivonne Santibáñez ${ }^{\mathrm{b}}$, Pablo Castillo ${ }^{\mathrm{a}}$, R. Mauricio Barría ${ }^{\mathrm{a}}$
}

aUnidad de Kinesiología, Instituto de Aparato Locomotor y Rehabilitación, Universidad Austral de Chile, Valdivia, Chile bEscuela de Kinesiología, Facultad de Medicina, Universidad Austral de Chile, Valdivia, Chile

Recibido: 12 de junio de 2019; Aceptado: 1 de diciembre de 2019

\section{¿Qué se sabe del tema que trata este estudio?}

Los trastornos respiratorios del sueño (TRS) se relacionan con la malnutrición por exceso, principalmente con el índice de masa corporal (IMC), y se asocian a un bajo rendimiento académico en escolares.

\section{¿Qué aporta este estudio a lo ya conocido?}

Se propone la pesquisa de TRS mediante un cuestionario costoefectivo que entrega prevalencia del trastorno. A nivel local, se determinó que los TRS se relacionan con el porcentaje de pliegues cutáneos (bicipital y tricipital), no así con el IMC.

\section{Resumen}

La malnutrición por exceso en la infancia constituye una epidemia mundial y se asocia a múltiples enfermedades y complicaciones. Entre ellas, destacan los trastornos respiratorios del sueño (TRS), espectro de enfermedades que han emergido como un problema de salud relevante. Objetivo: Evaluar la relación entre la composición corporal y la presencia de TRS en escolares. Sujetos y Método: Estudio observacional analítico de corte transversal en 127 escolares de primero a octavo básico escogidos aleatoriamente de cinco establecimientos municipales de Valdivia, Chile. La incorporación al estudio ocurrió luego del proceso de consentimiento informado del responsable del escolar y asentimiento informado del menor. Se realizaron mediciones antropométricas y se determinó la presencia de TRS mediante el Pediatric Sleep Questionnaire (PSQ). Para el análisis se aplicó estadística descriptiva, $\mathrm{t}$ de Student y test $\chi^{2}$ para determinar asociación de variables con la presencia de TRS. Resultados: Destacó alta prevalencia de malnutrición por exceso $(71,7 \%)$ y la obesidad alcanzó $39,4 \%$. En tanto la prevalencia de TRS fue 32,3\%. Hubo mayor proporción de niños con TRS en escolares obesos severos $(56,3 \%)$, así como una media significativamente mayor en niños con TRS para los pliegues bicipital $(14,6 \mathrm{~mm} \pm 7,3$ vs. $12,0 \mathrm{~mm} \pm 6,6 ; \mathrm{p}=0,002)$ y tricipital $(19,8 \pm 6,7 \mathrm{~mm}$ vs. $16,2 \mathrm{~mm} \pm 6,0$; $\mathrm{p}=0,04)$. Conclusiones: Existen altas prevalencias de malnutrición por exceso y TRS. De las medidas antropométricas, la presencia de TRS se asoció con mayor grosor de los pliegues bicipital y tricipital.
Palabras clave:

Síndromes de la apnea del sueño;

Obesidad;

Salud escolar;

Salud Infantil;

Encuestas y

Cuestionarios 


\begin{abstract}
Overnutrition in childhood constitutes a global epidemic and has been associated with multiple diseases and complications. Among them, sleep-disordered breathing (SDB) stands out, a spectrum of diseases that have emerged as a relevant health problem. Objective: To evaluate the association between nutritional status and SDB in schoolchildren. Subjects and Method: Cross-sectional analytical study of 127 schoolchildren randomly selected from five public schools in Valdivia, Chile. After the informed assent and informed consent process of the child and parents/guardian respectively, the students were incorporated into the study. Anthropometric measurements were performed and the presence of SDB was determined through the Pediatric Sleep Questionnaire (PSQ). For the data analysis, the t-test and $\chi^{2}$ test were used to determine the association of variables with SDB. Results: There was a high prevalence of overnutrition $(71.7 \%)$ and obesity reached $39.4 \%$. Regarding the prevalence of SDB, it was $32.3 \%$. There was a higher proportion of children with SDB in severely obese schoolchildren (56.3\%), as well as, a significantly higher mean of biceps and triceps skinfold thickness in children with SDB $(14.6 \mathrm{~mm} \pm 7.3$ vs. $12.0 \mathrm{~mm} \pm 6.6, \mathrm{p}=0.002$, and $19.8 \pm 6.7 \mathrm{~mm}$ vs. $16.2 \mathrm{~mm} \pm 6.0, \mathrm{p}=0.04$, respectively). Conclusions: There is high prevalence of overnutrition and SDB. Out of the anthropometric measurements, the presence of SDB was associated with greater thickness of the biceps and triceps skinfolds.
\end{abstract}

\section{Keywords:}

Sleep Apnea Syndromes; Obesity; School Health; Child Health; Surveys and questionnaires

\section{Introducción}

Los trastornos respiratorios del sueño (TRS) en niños, conforman un grupo de trastornos con un amplio espectro de presentación clínica y severidad, que incluye desde el ronquido primario hasta la apnea obstructiva del sueño, con consecuencias variables tales como la somnolencia diurna o comportamiento hiperactivo, u otras de mayor significación como dificultades en el aprendizaje y bajo rendimiento escolar ${ }^{1}$.

Estudios sobre TRS han demostrado las dificultades en su diagnóstico debido a la variedad de problemas metodológicos, la mayoría de los cuales implican heterogeneidad en la precisión diagnóstica. Se describen frecuencias de TRS informadas por padres en base a síntomas que varían entre 4 y $11 \%^{2}$. La prevalencia de síntomas de TRS encontrada en distintos países es diferencial, observándose alta prevalencia en Nigeria $^{3}$ $(34 \%)$ y Brasil $^{4}(27,6 \%)$ y menor en Malasia ${ }^{5}(14,9 \%)$ y Alemania $^{6}(10,1 \%)$. En población infantil chilena se ha reportado una prevalencia de $18 \%$ de roncador habitual, como representación inicial del espectro de TRS ${ }^{7}$.

El estándar de oro para el diagnóstico de los TRS es la polisomnografía (PSG) $)^{8}$, estudio que entrega información sobre parámetros neurofisiológicos y cardiorrespiratorios, siendo un examen de alto costo que necesita ser aplicado en laboratorios del sueño, lo que dificulta su acceso. Es por esto que para facilitar la pesquisa de TRS se han desarrollado cuestionarios pediátricos, de bajo costo y fáciles de realizar. Uno de ellos es el Pediatric Sleep Questionnaire (PSQ), que incluye 22 preguntas sobre los síntomas de TRS y que es aplicado a padres o cuidadores?.

Existe distinta evidencia sobre la relación de obesi- dad, apneas y arquitectura del sueño en escolares; sin embargo, parte importante de los resultados de éstas apuntan al desarrollo de disfunciones metabólicas y morbilidad asociada ${ }^{10}$.

La Región de los Ríos, contexto de desarrollo de este estudio, es una de las regiones con mayor índice de obesidad en Chile. Según datos del año 2015 se constató $45 \%$ de escolares de octavo básico con un índice de masa corporal (IMC) que los clasificó en condición de sobrepeso (20\%) u obesidad $(25 \%)$, cifras que van aumentando paulatinamente con el transcurso del tiempo ${ }^{11}$.

Por otro lado, ya se ha demostrado la relación entre los TRS y el menor rendimiento académico ${ }^{1,12}$, lo que enfatiza el desarrollo de este estudio en población escolar. Consecuentemente, este trabajo tuvo como objetivo evaluar la relación entre composición corporal y la presencia de TRS en escolares de primero a octavo básico pertenecientes a establecimientos educacionales municipales de la ciudad de Valdivia, Chile.

\section{Sujetos y Metodo}

\section{Diseño}

Estudio observacional analítico de corte transversal orientado a establecer la relación entre el estado nutricional y presencia de TRS. El trabajo de campo se desarrolló durante el mes de diciembre de 2016. La evaluación y aprobación de este estudio se llevó a cabo por el Comité Ético Científico del Servicio de Salud de Valdivia (Ord. $N^{\circ} 449,29$ de noviembre de 2016) y la participación de cada niño fue autorizada por sus padres y/o responsable a través de consentimiento informado sumado al asentimiento del menor. 


\section{Población objetivo y muestra}

La muestra estuvo compuesta por escolares de ambos sexos, de primero a octavo básico pertenecientes a cinco establecimientos municipales de la ciudad de Valdivia, Chile, escogidos por muestreo aleatorio simple. Dentro de los establecimientos la selección de escolares se realizó a través de un muestreo aleatorio estratificado con afijación proporcional. Se excluyeron aquellos niños que presentaban alguna afección neuromotora o respiratoria lo cual fue constado por registros de salud y reporte de los padres (ej: asma). De una población total de 2.660 escolares pertenecientes a establecimientos municipales de la ciudad, se calculó una muestra representativa de 209 estudiantes considerando un nivel de confianza 95\%, una precisión (error de estimación) de 5\% y una proporción esperada de TRS en la población de $18 \%$. Se utilizó para el cálculo de tamaño de muestra el programa nQuery Advisor ${ }^{\circledR} 7.0$ (Statistical Solutions, Saugus, Mass., USA).

No obstante lo anterior, se contó con la aprobación de 191 padres o apoderados. Además, 51 potenciales participantes no asistieron el día de la evaluación y 13 no contestaron el cuestionario PSQ, quedando finalmente una muestra de 127 escolares.

\section{Técnica de recolección de datos y procedimientos}

Las evaluaciones se llevaron a cabo en las instalaciones de cada establecimiento. En primer lugar, se realizaron las mediciones antropométricas, y luego se aplicó el cuestionario PSQ a los padres o responsable del escolar.

Las variables antropométricas evaluadas fueron peso, talla, pliegue bicipital, tricipital, supraespinal y subescapular, además del perímetro de cuello y de cintura. Se calculó el IMC dividiendo el peso por la talla al cuadrado $\left(\mathrm{IMC}=\right.$ peso $(\mathrm{kg}) /$ talla $\left.(\mathrm{cm})^{2}\right)$ y se clasificó como bajo peso, normopeso, sobrepeso y obesidad/ obesidad severa según la Norma para evaluación nutricional del Ministerio de Salud de Chile ${ }^{13}$. Los pliegues fueron medidos con Caliper-Adipómetro Slim Guide ${ }^{\varpi}$. La composición de grasa corporal se determinó mediante la fórmula de Lohman específica para niños por sexo y edad ${ }^{14}$, para lo cual se calculó previamente la Densidad (D) mediante las ecuaciones de Brook para niños y niñas hasta 11 años y de Durnin y Womersley de 12 años en adelante ${ }^{15}$.

Además la recogida de datos incluyó información demográfica como edad y sexo, y académicas como curso y rendimiento académico operacionalizado como promedio global de calificaciones (en escala de 1 a 7). No fue posible obtener datos separados por ámbitos o materias.

\section{Cuestionario PSQ}

El cuestionario de los trastornos respiratorios re- lacionados con el sueño PSQ, versión reducida en español, se utilizó para los propósitos del estudio. Este instrumento, con buena precisión diagnóstica y utilizado en Chile ${ }^{16}$, contiene 22 preguntas divididas en tres apartados: A) Conducta durante la noche y mientras duerme; B) Conducta durante el día y otros problemas posibles; y C) Síntomas del trastorno por déficit de atención/hiperactividad. Las posibles respuestas y puntuaciones para cada ítem de los apartados A y B son "Sî" = 1, "No" y "No sabe" = 0. Para el apartado "C", para reducir las respuestas a los formatos Sí/No en los elementos con más respuestas posibles, "Nunca" y "Algunas veces" también se calificó como "No" $=0$, mientras que "Muchas veces" y "Casi siempre" se calificaron como "Sî"=1. El puntaje PSQ se calculó sumando el recuento total de todas las respuestas "Sí"/ total de todas las respuestas "No" y "Sí". Se utilizó el valor de corte $\geq 0,33^{16}$.

\section{Análisis estadístico}

Los datos obtenidos fueron almacenados en una planilla del programa Microsoft Excel 2013 y analizados usando el programa Stata v.11.2 (StataCorp LP, College Station, Texas, USA, 2009). En primer lugar se empleó estadística descriptiva para caracterizar a los escolares. Inicialmente se evaluó el ajuste a la normalidad de las variables cuantitativas analizando su distribución gráfica (histograma) y mediante el test de Kolmogorov-Smirnov. De esta forma, las variables cuantitativas como edad, promedio de calificaciones y las distintas medidas antropométricas se describen como media y desviación estándar. En tanto las cualitativas, como sexo y curso, se muestran como distribución de frecuencias absolutas y relativas. Para evaluar posibles asociaciones entre la variable TRS y las variables antropométricas y rendimiento académico se aplicó la prueba de la T ( $t$ de Student). Para comparar presencia de TRS según categorías de estado nutricional se empleó la prueba $\chi^{2}$. Se consideró un nivel de significancia estadística con valor de $\mathrm{p}<0,05$.

\section{Resultados}

Los niños estudiados se caracterizaron por ser en su mayoría hombres $(68,5 \%)$ con una edad media ( $\pm \mathrm{DE})$ de 9,4 años $( \pm 2,3)$. La distribución por cursos mostró mayor concentración en segundo ciclo de enseñanza básica $(51,2 \%)$. Respecto del rendimiento académico, se observó un promedio de calificaciones de 6,01 $\pm 0,49$, oscilando entre 4,1 y 6,8 (tabla 1 ).

De las variables antropométricas (tabla 2), destacó un IMC medio de 21,1 $\pm 4,2$, a partir de lo cual se determinó que un 71,7\% presentaba malnutrición por exceso. La obesidad alcanzó 39,4\%. No hubo diferen- 


\section{Tabla 1. Características demográficas y académicas}

\begin{tabular}{lcc}
\hline Variable & & \\
\hline Edad (años); media \pm DE & 9,4 & 2,3 \\
Sexo n, \% & & \\
$\quad$ Mujer & 40 & 31,5 \\
$\quad$ Hombre & 87 & 68,5 \\
Curso & & \\
$\quad 1$ - 4 (primer ciclo básico) & 62 & 48,8 \\
$\quad 5$ - 8 (segundo ciclo básico) & 65 & 51,2 \\
Promedio de calificaciones media \pm DE & 6,01 & 0,49 \\
\hline
\end{tabular}

DE: desviación estándar.

Tabla 2. Características antropométricas

\begin{tabular}{|c|c|c|c|c|}
\hline Características antropométricas & Media & $\mathrm{DE}$ & Min. & Máx. \\
\hline Circunferencia de cuello $(\mathrm{cm})$ & 30,5 & 3,3 & 23,5 & 40 \\
\hline Circunferencia de cintura $(\mathrm{cm})$ & 69,6 & 11,2 & 46 & 107 \\
\hline Pliegue supraespinal (mm) & 15,3 & 8,0 & 3 & 42 \\
\hline Pliegue bicipital (mm) & 12,8 & 6,9 & 2 & 35 \\
\hline Pliegue tricipital (mm) & 17,4 & 6,4 & 5 & 37 \\
\hline Pliegue subescapular (mm) & 13,6 & 7,2 & 3 & 35 \\
\hline Índice de masa corporal $\left(\mathrm{kg} / \mathrm{m}^{2}\right)$ & 21,1 & 4,2 & 14,1 & 38,4 \\
\hline \multirow[t]{2}{*}{ Porcentaje de grasa (\%) } & 24,2 & 7,6 & 2,9 & 40,6 \\
\hline & $\mathrm{n}$ & & $\%$ & \\
\hline \multicolumn{5}{|l|}{ Estado nutricional } \\
\hline Bajo peso & 1 & & 0,8 & \\
\hline Normopeso & 35 & & 27,5 & \\
\hline Sobrepeso & 41 & & 32,3 & \\
\hline Obesidad/Obesidad severa & 50 & & 39,4 & \\
\hline
\end{tabular}

DE: desviación estándar; Min.: valor mínimo; Máx.: valor máximo.

Tabla 3. Distribución de TRS por Estado Nutricional

\begin{tabular}{|c|c|c|c|c|}
\hline \multirow[t]{2}{*}{ Estado nutricional } & \multicolumn{2}{|c|}{$\begin{array}{l}\text { TRS (-) } \\
n=86 \\
\end{array}$} & \multicolumn{2}{|c|}{$\begin{array}{l}\text { TRS (+) } \\
n=41\end{array}$} \\
\hline & $\mathrm{n}$ & $\%$ & $n$ & $\%$ \\
\hline Bajo peso & 0 & 0 & 1 & 2,4 \\
\hline Normopeso & 25 & 29,1 & 10 & 24,4 \\
\hline Sobrepeso & 32 & 37,2 & 9 & 21,9 \\
\hline Obesidad/Obesidad severa & 29 & 33,7 & 21 & 51,2 \\
\hline Total & 86 & 100 & 41 & 100 \\
\hline
\end{tabular}

TRS: trastorno respiratorio del sueño. $p=0,070$ Test $\chi^{2}$. cias significativas por sexo tanto en IMC como en distribución por estado nutricional.

La prevalencia de TRS fue $32,3 \%$ y afectó a $22,5 \%$ de las mujeres y $36,8 \%$ de los hombres, diferencia que, si bien marca una tendencia, no fue significativa $(\mathrm{p}=0,152)$. Tampoco hubo asociación significativa con la edad, observándose edades medias similares entre niños con y sin TRS $(9,2 \pm 2,4$ vs $9,5 \pm 2,2$, respectivamente; $\mathrm{p}=0,4772$ ).

La proporción de TRS por categorías de estado nutricional (tabla 3 ) mostró diferencias no significativas $(\mathrm{p}=0,070)$. En esto se observó que 51,2\% de los niños con TRS se encontraron en la categoría de obesidad/ obesidad severa en tanto en los niños sin TRS esta proporción alcanzó $33,7 \%$.

En la evaluación de los pliegues cutáneos según condición de TRS, se observó una media significativamente mayor en escolares con TRS para la medición de pliegues bicipital $(14,6 \pm 7,3$ vs $12,0 \pm 6,6 ; p=0,0029)$ $y$ tricipital $(19,8 \pm 6,7$ vs $16,2 \pm 6,0 ; p=0,0497)$ (figura $1)$. El resto de las mediciones de pliegues y circunferencias no mostraron diferencias según condición de TRS.

Al comparar el porcentaje de grasa por condición de TRS (figura 2) se observó una media mayor en niños con el trastorno (23,3 vs 26,$0 ; p=0,05)$.

Por último, al analizar el rendimiento académico (promedio global de calificaciones) no se detectaron diferencias significativas al comparar niños con y sin TRS $(6,0 \pm 0,45$ vs $6,0 \pm 0,51 ; \mathrm{p}=0,74)$.

\section{Discusión}

Este estudio ha permitido comprobar la alta prevalencia de TRS y de malnutrición por exceso donde destaca la proporción elevada de obesidad en escolares de enseñanza básica de establecimientos municipales de Valdivia.

La prevalencia de TRS encontrada (32,3\%) supera la reportada previamente en un estudio chileno efectuado en diferentes comunas del país y que alcanzó una prevalencia global de $17,7 \%{ }^{17}$. No obstante, en dicho estudio, se encontraron prevalencias tan elevadas como las registradas en nuestro trabajo en comunas del sur como Porvenir $(33,3 \%)$ y Puerto Natales $(36,4 \%)$. En otro estudio nacional realizado en Concepción, Chile, se registró una prevalencia elevada de $24,6 \%{ }^{18}$. Se determinó mayor proporción de TRS en hombres que en mujeres al igual que lo informado en otros estudios chilenos ${ }^{17}$ y extranjeros ${ }^{5}$.

Dentro de los niños que presentaron TRS, un alto porcentaje era obeso $(51,2 \%)$, sin embargo, si bien hubo una tendencia, no se encontró asociación significativa entre estas variables, a diferencia del estudio de Sánchez et al. ${ }^{17}$ en el cual fue significativa la presencia de niños con obesidad severa en el grupo con TRS. 

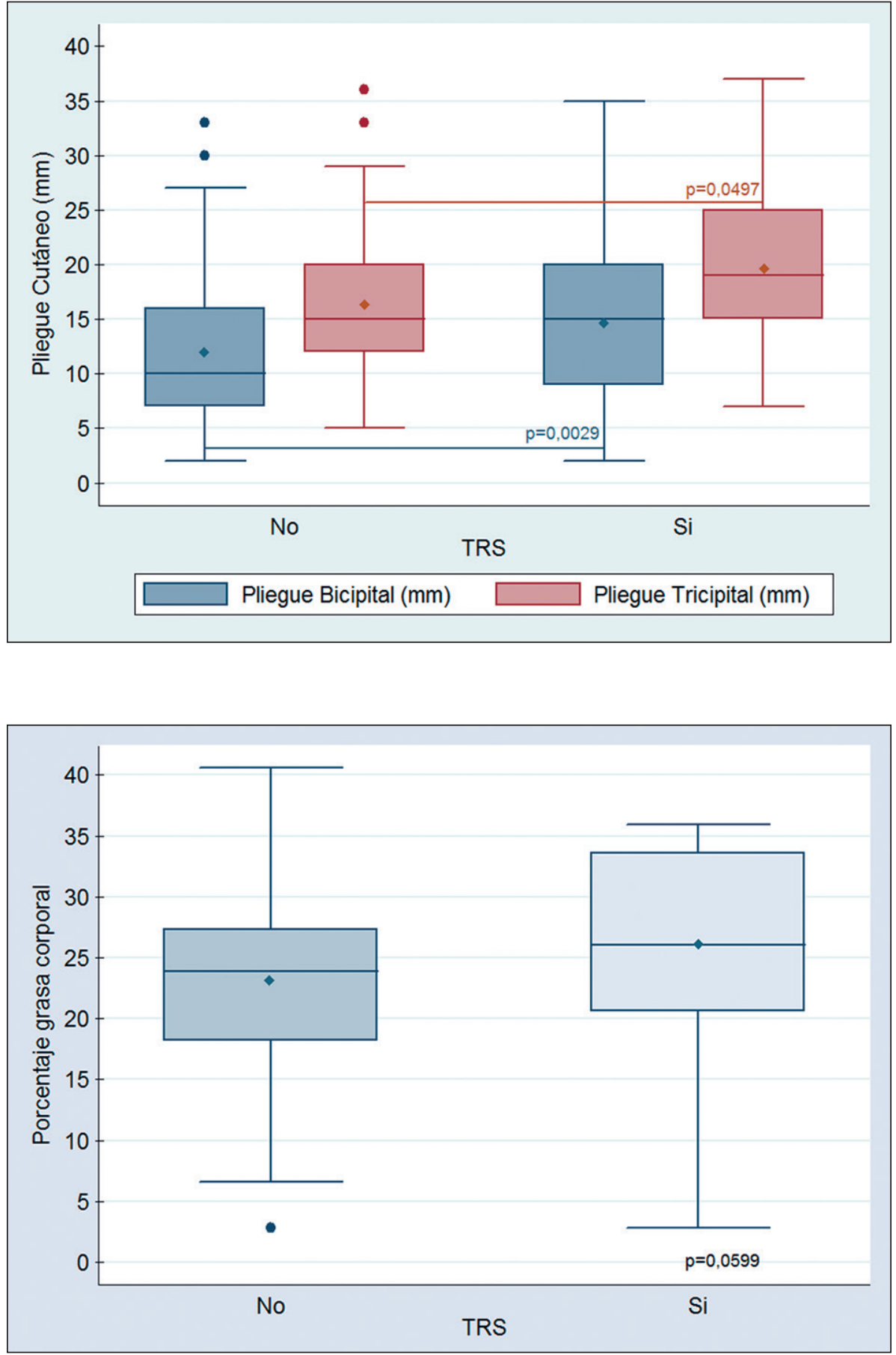

Figura 1. Pliegues cutáneos bicipital y tricipital según condición de TRS.
Figura 2. Porcentaje de grasa según condición de TRS.
La evidencia disponible sugiere que la obesidad constituye un factor de riesgo de TRS en niños producto de mecanismos fisiopatológicos que incluyen cierre de las vías respiratorias, tendencia al colapso por infiltración de grasa en las estructuras de la vía aérea superior y afección de la ventilación, lo que favorecería la aparición de anomalías en la respuesta ventilatoria ${ }^{19}$. Además, los niños con obesidad y TRS tienen mayor probabilidad de que su condición persista o empeore en el tiempo presentando en la adolescencia una distribución de grasa central e incremento de la adiposidad visceral ${ }^{20}$. La relación de obesidad y TRS, además tiene impacto sobre la calidad de vida en la infancia dado que se ha observado que niños con obesidad y TRS muestran peor calidad de vida comparado con niños solo con obesidad o TRS o niños sanos ${ }^{21}$.

Se constató una relación significativa entre la presencia de TRS e indicadores antropométricos como 
el pliegue bicipital y tricipital, resultados que difieren de lo encontrado en el estudio realizado en Malasia en que no se verificó esta relación para el pliegue bicipital, pero sí una asociación univariada con circunferencia de cuello y cintura ${ }^{5}$.

La adiposidad se ha descrito como un importante factor de riesgo para el desarrollo de TRS ${ }^{22,23}$, especialmente el incremento de grasa visceral que ha determinado mayor severidad del cuadro ${ }^{24}$. El mecanismo por lo que la adiposidad predispone a TRS se vincula a alteraciones en la estructura y función de los músculos respiratorios, reducción de la compliance de la pared torácica, y deterioro de la capacidad funcional residual ${ }^{25}$.

En cuanto al rendimiento académico, a diferencia de estudios internacionales y nacionales previos que han determinado una clara asociación entre TRS y un peor desempeño escolar representado a través de distintas medidas de habilidades o dominios de aprendizaje $^{18,26-28}$, este trabajo no logró demostrar diferencias globales de rendimiento entre niños con y sin TRS. Sin embargo, como ya se comentó, la medición de rendimiento fue definida de manera global y no separada por materias en lo que sí podría esperarse una asociación específica. Por otro lado, no se analizó focalizadamente la ejecución de funciones cognitivas y académicas con baterías de evaluaciones específicas.

Algunas limitaciones de este trabajo deben ser comentadas para valorar con cautela estos resultados. En primer lugar, la pesquisa de TRS se basó en un cuestionario, el cual, a pesar de ser muy utilizado y con alta fiabilidad, no constituye el estándar de oro para identificar TRS, como si lo es la PSG. Por otro lado, como se informó, el tamaño de muestra alcanzó sólo un 60,8\% del estipulado en la propuesta del estudio. Por lo anterior, la falta de asociación entre variables que en otras investigaciones ha sido encontrada pudo obedecer a una falta de potencia estadística. En el caso particular del rendimiento académico, y aunque no era el foco del estudio, la medición global de esta variable a través del promedio global de calificaciones puede estar enmascarando una asociación separada por materias o áreas.

Por último, hubo variables que no estuvieron disponibles para ser incorporadas en el análisis dada la homogeneidad de la muestra al provenir todos los participantes de colegios municipales, por ejemplo, el nivel socioeconómico, el que también ha sido un factor relacionado en otros trabajos al desarrollo de TRS.

En conclusión, en este trabajo se han constatado altas prevalencias de malnutrición por exceso y TRS. De las medidas antropométricas, la presencia de TRS se asoció con mayor grosor de los pliegues bicipital y tricipital, confirmando que son componentes que deben ser considerados en la evaluación de los escolares.

\section{Responsabilidades Éticas}

Protección de personas y animales: Los autores declaran que los procedimientos seguidos se conformaron a las normas éticas del comité de experimentación humana responsable y de acuerdo con la Asociación Médica Mundial y la Declaración de Helsinki.

Confidencialidad de los datos: Los autores declaran que han seguido los protocolos de su centro de trabajo sobre la publicación de datos de pacientes.

Derecho a la privacidad y consentimiento informado: Los autores han obtenido el consentimiento informado de los pacientes y/o sujetos referidos en el artículo. Este documento obra en poder del autor de correspondencia.

\section{Conflicto de intereses}

Los autores declaran no tener conflicto de intereses.

\section{Referencias}

1. Brockmann PE, Bertrand P, Pardo T, et al. Prevalence of habitual snoring and associated neurocognitive consequences among Chilean school aged children. Int J Pediatr Otorhinolaryngol 2012;76:132731.

2. Lumeng JC, Chervin RD. Epidemiology of pediatric obstructive sleep apnea. Proc Am Thorac Soc 2008;5:242-52.

3. Alabi BS, Abdulkarim AA, Musa IO, et al. Prevalence of snoring and symptoms of sleep disordered breathing among primary school pupils in Ilorin, Nigeria. Int J Pediatr Otorhinolaryngol
2012;76:646-8.

4. Petry C, Pereira MU, Pitrez PM, et al. The prevalence of symptoms of sleep-disordered breathing in Brazilian schoolchildren. J Pediatr (Rio J) 2008;84:123-9.

5. Fadzil Abdullah AA, Jamalludin AR, Norrashidah AW, et al. Prevalence of sleep disordered breathing symptoms among Malay school children in a primary school in Malaysia. Med J Malaysia 2012;67:181-5.

6. Urschitz MS, Guenther A, Eitner S, et al. Risk factors and natural history of habitual snoring. Chest 2004;126: 790-800.
7. Pardo T, Holmgren NL, Cerda J, et al. Prevalencia disímil de trastornos respiratorios del sueño en escolares. Rev Chil Pediatr 2013;84:145-51.

8. Stehling F, Keull J, Olivier M, et al. Validation of the screening tool ApneaLink(R) in comparison to polysomnography for the diagnosis of sleep-disordered breathing in children and adolescents. Sleep Med 2017;37:13-8.

9. Vila MT, Miralles Torres A, Beseler Soto B. Versión española del Pediatric Sleep Questionnaire. Un instrumento útil en la investigación de los trastornos del sueño en la infancia. Análisis de su fiabilidad. An Pediatr (Barc) 2007;66:121-8. 
10. Koren D, Gozal D, Philby MF, et al. Impact of obstructive sleep apnoea on insulin resistance in nonobese and obese children. Eur Respir J 2016;47:115261.

11. Agencia de Calidad de la Educación. Estudio Nacional de Educación Física 2015. [Internet]. 2016. Disponible en http://www.revistadeeducacion. cl/wp-content/uploads/2016/10/ PRESENTACION-ED-FISICA.pdf.

12. Brockmann PE, Urschitz MS, Schlaud $\mathrm{M}$, et al. Primary snoring in school children: prevalence and neurocognitive impairments. Sleep Breath 2012;16:23-9.

13. Ministerio de Salud. Subsecretaría de Salud Pública. Norma para la evaluación nutricional de niños, niñas y adolescentes de 5 a 19 años de edad. Santiago: Ministerio de Salud. 2016.

14. Lohman TG. Applicability of body composition techniques and constants for children and youths. Exerc Sport Sci Rev 1986;14:325-57.

15. Martínez Sopena MJ, Redondo Del Río MP, Alonso Franch M. Valoración estado nutricional del obeso: estimación de la masa grasa. Bol Pediatr 2006;46:275-91.

16. Bertran K, Mesa T, Rosso K, et al. Diagnostic accuracy of the Spanish version of the Pediatric Sleep Questionnaire for screening of obstructive sleep apnea in habitually snoring children. Sleep Med 2015;16:631-6.

17. Sánchez T, Rojas C, Casals M, et al. Trastornos respiratorios del sueño en niños escolares chilenos: prevalencia y factores de riesgo. Rev Chil Pediatr 2018;89:718-25.

18. Gatica D, Rodriguez-Nunez I, Zenteno $\mathrm{D}$, et al. Association between sleeprelated breathing disorders and academic performance among children from Concepcion, Chile. Arch Argent Pediatr 2017;115:497-500.

19. Esteller-Moréa E, Castells-Vilella L, Segarra-Isernc F, et al. Obesidad infantil y trastornos respiratorios del sueño. Acta Otorrinolaringol Esp 2012;63:180-6.

20. Frye SS, Fernández-Mendoza J, Calhoun SL, et al. Childhood obesity, weight loss and developmental trajectories predict the persistence and remission of childhood sleep-disordered breathing. Pediatr Obes 2019;14.

21. Katz SL, MacLean JE, Barrowman N, et al. Long-Term Impact of Sleep-Disordered Breathing on Quality of Life in Children With Obesity. J Clin Sleep Med 2018;14:451-8.

22. Verhulst SL, Schrauwen N, Haentjens $\mathrm{D}$, et al. Sleep-disordered breathing in overweight and obese children and adolescents: prevalence, characteristics and the role of fat distribution. Arch Dis Child 2007;92:205-8.

23. Verhulst SL, Van Gaal L, De Backer W, et al. The prevalence, anatomical correlates and treatment of sleep-disordered breathing in obese children and adolescents. Sleep Med Rev 2008;12:33946.

24. Bixler EO, Fernandez-Mendoza J, Liao D, Calhoun S, Rodriguez-Colon SM, Gaines J, et al. Natural history of sleep disordered breathing in prepubertal children transitioning to adolescence. Eur Respir J 2016;47:1402-9.

25. Kohler MJ, van den Heuvel CJ. Is there a clear link between overweight/obesity and sleep disordered breathing in children? Sleep Med Rev 2008;12:347-61.

26. Gottlieb DJ, Chase C, Vezina RM, et al. Sleep-disordered breathing symptoms are associated with poorer cognitive function in 5-year-old children. J Pediatr 2004;145:458-64.

27. Bourke R, Anderson V, Yang JS, et al. Cognitive and academic functions are impaired in children with all severities of sleep-disordered breathing. Sleep Med 2011;12:489-96.

28. Galland B, Spruyt K, Dawes P, et al. Sleep disordered breathing and academic performance: a meta-analysis. Pediatrics 2015;136(4):e934-46. 